\title{
Monitoring herd incidence of intramammary infection in lactating cows using repeated longitudinal somatic cell count measurements
}

\author{
S. Dufour ${ }^{*}{ }^{1}$ and I. R. Dohoo* $\dagger$ \\ *Department of Health Management, Atlantic Veterinary College, University of Prince Edward Island, 550 University Ave., Charlottetown, \\ C1A 4P3, Canada \\ †Canadian Bovine Mastitis Research Network, CP 5000, St-Hyacinthe, Quebec, J2S 7C6, Canada
}

\begin{abstract}
The objective of the study was to evaluate the ability of an estimate of the herd intramammary infection (IMI) incidence rate computed using repeated somatic cell count (SCC) measurements (quarter- and composite-SCC; hereafter, the SCC-derived herd IMI incidence, SCCI) to predict the incidence rate computed using repeated quarter-milk bacteriological culture (hereafter, bacteriological culture incidence, BCI) during the lactating period. A cohort of 91 Canadian dairy herds was followed in 2007 and 2008. In each herd and at each of 4 sampling periods, a series of 3 to 7 quarter-milk samples was collected from a sample of 15 cows. Routine milk bacteriological culture was conducted to identify IMI, SCC was measured on the quarter-milk samples, and composite-SCC of the preceding and following dairy herd improvement (DHI) tests were obtained. Mastitis pathogens were grouped in 3 categories: major, minor, and any pathogens. For each herd and for each period, BCI was computed for each group of organisms. Similarly, SCCI were computed using quarter- and DHI composite-SCC and using a threshold of 200,000 cells/ $\mathrm{mL}$ to define infected quarters or cows. A linear regression model taking into account the structure of the data was used to compare the SCCI to the BCI. A similar model was used to compare fluctuations (i.e., changes from one sampling period to the next) over time of the SCCI and BCI. Measures of correlation between observed and predicted rates were computed and limits of agreement plots sketched to better explore the predictive ability of the SCCI. The quarter-milk SCC measurements that could be obtained-for instance, using on-line milking system measurements - appeared to be particularly valuable. Quarter-SCCI showed a positive and significant association with the BCI. However, limits of agreement plots indicated important disagreement for the small proportion of observations with very high BCI. Quarter-level SCCI and BCI fluctuations were
\end{abstract}

Received July 3, 2012.

Accepted December 8, 2012.

${ }^{1}$ Corresponding author: simon.dufour@umontreal.ca also significantly associated, and a substantial correlation (Spearman rho ranging from 0.54 to 0.58 ) could be seen between observed and predicted rates. Conversely, the predictive value of composite-DHI SCC for monitoring IMI incidence during the lactation seemed to be quite limited. Composite SCCI was strictly associated with major IMI BCI, showed a relatively low correlation with the observed rate (Spearman rho: 0.14), and was of little help for longitudinal monitoring of the IMI incidence.

Key words: lactating period, intramammary infection, incidence, somatic cell count

\section{INTRODUCTION}

Mastitis is one of the most costly diseases for the dairy industry worldwide and most of the economic loss is actually due to subclinical mastitis, a nonclinical inflammation of the udder usually resulting from bacterial IMI (Halasa et al., 2007). The prevalence of subclinical mastitis in a herd is directly determined by the rate at which new IMI (NIMI) are acquired and eliminated (i.e., the incidence and elimination rates; Schukken et al., 2003). In 2 recent studies conducted in Canada, a given variation of the incidence rates of Staphylococcus aureus and CNS IMI - the 2 pathogens most frequently recovered from apparently normal milking quarters in the country - was found to have a greater effect on IMI prevalence than a comparable variation of their elimination rates (Dufour et al., 2012a,b). These results confirm that, as for many other infectious diseases, preventing new infections may be the key to long-term control of subclinical mastitis. Reducing the incidence of IMI should, therefore, be the main objective of a subclinical mastitis control program, and frequent monitoring of the herd IMI incidence rate should, consequently, be an essential component of routine udder health surveillance.

Obtaining a valid estimate of the herd IMI incidence rate is quite difficult, however, because of the repeated IMI measurements needed to identify NIMI occurrence. In research settings, IMI incidence measurements are 
often obtained using longitudinal study designs involving repeated cow or quarter bacteriological milk culture (Zadoks et al., 2001; Dufour et al., 2012a,b). Conducting repeated milk bacteriological cultures on a regular basis to monitor the herd IMI incidence rate, however, is certainly not very practical for routine udder health monitoring of commercial dairy herds. Conversely, monthly composite SCC measurements are available on most dairy farms and could potentially be used as a proxy for NIMI to estimate herd IMI incidence rate. Furthermore, novel milking systems that can measure quarter SCC at milking could be used to generate the data needed to estimate and monitor herd IMI incidence rate.

Several SCC thresholds and absolute or relative SCC increases have been evaluated for their ability to accurately detect the occurrence of NIMI at cow level. An SCC-derived NIMI definition, consisting of a composite SCC going from under to over 200,000 cells/mL over a 28-d interval, has also been suggested (Dohoo and Leslie, 1991). Even though the sensitivity of the proposed threshold to identify occurrence of NIMI at the cow level was fairly low (32\%), it was, nonetheless, proposed as a useful criterion to compute herd-level incidence estimates and to monitor herd-level fluctuations in udder health status (Erskine, 2001). Today, the herd incidence rate derived from individual cow monthly DHI testing is reported by many DHI organizations and this measure is routinely monitored by dairy practitioners and extension agents to evaluate herd performances and to help decide on clinical investigations and interventions. Furthermore, such a measure of the herd IMI incidence has been used in research settings and variation of this parameter in large populations of dairy farms has been investigated to set targets for mastitis control programs (Lukas et al., 2005; Valde et al., 2005; Madouasse et al., 2010). Finally, some authors have recommended goals for SCC-derived herd IMI incidence (SCCI) and have suggested potential interpretations and course of investigation for herds exceeding these goals (Schukken et al., 2003; Bradley and Green, 2005).

Despite the widespread use of SCCI estimates, little is known about the relationship between these and bacteriological culture-derived herd IMI incidence (BCI) estimates. In the original study investigating the accuracy of different SCC-derived NIMI definitions, a strong correlation was found between SCCI and BCI rates (Dohoo and Leslie, 1991). In that study, however, the same data were used for selection of the criterion and for its subsequent evaluation, which may have biased the analysis presented, as acknowledged by the authors, and may have led to an inappropriately high correlation estimate. Likewise, little is known about the relationship between a given change over time in the SCCI rate and the behavior of its bacteriological culture counterpart. Consequently, despite all the proposed targets, little is known about the suitability of SCCI estimates when used as a proxy for BCI or as a tool for detecting undesirable fluctuations of herd IMI incidence over time. In both instances this may lead to inappropriate clinical investigations and interventions.

The main objective of this study was to evaluate the predictive value of herd IMI incidence density rate computed from repeated SCC measurements (quarterand composite-SCC measurements) as a proxy for the herd IMI incidence density rate that would be obtained from repeated bacteriological quarter-milk cultures. A secondary objective was to evaluate the predictive value of SCCI density rate fluctuations over time as a surrogate to BCI density rate fluctuations for longitudinal monitoring of the herd IMI incidence rate.

\section{MATERIALS AND METHODS}

In 2006, 91 Canadian dairy herds were recruited to participate to the National Cohort of Dairy Farms (NCDF) of the Canadian Bovine Mastitis Research Network (CBMRN). A complete description of the herd selection process and of the general characteristics of these herds has been published elsewhere (Reyher et al., 2011). Briefly, herds were recruited in 4 regions of Canada based on willingness of the dairy producer to participate in a 2-yr (2007-2008) cohort study.

At the beginning of each of 4 intensive sampling periods (March-May 2007, June-August 2007, JanuaryMarch 2008, and June-August 2008), a sample of 15 apparently normal milking cows were selected in each NCDF herd. Five of these cows were the most recently calved cows and the remaining 10 cows were randomly chosen within the lactating cows that were expected to remain in the milking herd for at least 2 mo. During the March-May 2007, January-March 2008, and June-August 2008 sampling periods, series of 3 single quarter-milk samples were collected from the selected cows at intervals of 3 wk. During the June-August 2007 sampling period, series of 7 single quarter-milk samples were collected at weekly intervals. The more intensive sampling period in summer 2007 was primarily carried out for another CBMRN study evaluating definitions of IMI (Dohoo et al., 2011), but was also used in the current study to maximize the quality of the data available.

Milk samples were frozen for storage and later thawed and cultured using a standardized protocol based on the National Mastitis Council guidelines for culture and species identification (Hogan et al., 1999). Briefly, $10 \mu \mathrm{L}$ of milk was streaked on a Columbia agar $+5 \%$ sheep blood plate and incubated aerobically at 
$35^{\circ} \mathrm{C}$ for $24 \mathrm{~h}$. The phenotypically different types of colonies were then enumerated and speciated using recommended bacteriological procedures (Hogan et al., 1999) and reincubated for an additional $24 \mathrm{~h}$. Quarter somatic cell count measurements were then obtained for each milk sample using the Fossomatic cell counter (Fossomatic 4000 series; Foss Electric A/S, Hillerød, Denmark). Dairy herd improvement programs data for the study period were retrieved for each participating herd to obtain monthly individual cow composite SCC measurements of the selected cows. Somatic cell count measurements were, therefore, available as quarterlevel SCC (CBMRN data) and as composite-level SCC (DHI data).

\section{Definitions for Bacteriological Culture IMI, NIMI, and Incidence Rate}

IMI. Milk samples for which $\geq 3$ phenotypically different species were cultured were considered contaminated and uninformative unless Staph. aureus or Streptococcus agalactiae colonies were identified. Based on recommendations from Dohoo et al. (2011), presence of an IMI in a quarter on the day that an individual milk sample was collected was defined as recovery of a given organism in concentration $\geq 100 \mathrm{cfu} / \mathrm{mL}$ of milk in the associated sample - with the exception of CNS organisms, for which $\geq 200 \mathrm{cfu} / \mathrm{mL}$ of milk was required. Intramammary infections were further collapsed in 3 broader categories: major IMI, minor IMI, and any IMI. Presence of a major IMI was defined as presence of an IMI caused by Staph. aureus, Strep. agalactiae, Streptococcus dysgalactiae, Streptococcus uberis, Escherichia coli, or Klebsiella spp. Presence of a minor IMI was defined as presence of an IMI caused by CNS or Corynebacterium spp. Finally, presence of any IMI was defined as the presence of an IMI caused by any staphylococci, streptococci, or gram-negative species, or the presence of an IMI caused by Corynebacterium spp., Enterobacter spp., Trueperella pyogenes, Nocardia spp., Prototheca spp., yeast, or fungi.

NIMI. To define NIMI, series of milk samples collected at 3-wk intervals during most periods and at weekly intervals during June-August 2007 were used. A quarter was only considered at risk of acquiring a NIMI on a given day if the 2 preceding milk samples of the series yielded negative results for the group of organisms under investigation. Quarters with $<2$ available negative samples before the day of interest were not considered at risk of NIMI and, therefore, did not contribute to the herd incidence rate calculation (i.e., 0 NIMI and $0 \mathrm{~d}$ at risk). Whenever observed, NIMI were deemed to have occurred halfway between the collection of the preceding negative sample and the following positive sample. Table 1 illustrates the computation of NIMI and days at risk for the 3 -wk and 1-wk intervals sampling periods. As can be seen from Table 1, during the 3 -wk intervals periods, only series constituted of negative, negative, negative and of negative, negative, positive samples contributed to the incidence calculation. During the weekly sampling period (June-August 2007), only series with $\geq 2$ subsequent negative samples could contribute to the herd IMI incidence calculation.

Incidence Rates. Quarter BCI were computed for each herd and at each of the sampling periods as follows: for a given herd and during a given sampling period, the total number of NIMI was computed and divided by the total number of quarter-days at risk of NIMI acquisition. This rate was then multiplied by 30.5 to report BCI in NIMI/quarter-month.

\section{Definitions for SCC-Derived NIMI and Incidence Rate}

To define NIMI and herd IMI incidence based on SCC measurements (referred to as NIMI SCC and SCCI respectively) using quarter-level SCC, SCC results from samples 1 and 3 of each series of 3 milk samples were retained (i.e., samples 1 and 3 for the March-May 2007, January-March 2008, and the JuneAugust 2008 sampling periods; samples 1 and 3, 2 and 4, 3 and 5, 4 and 6 , and 5 and 7 for the June-August 2007 sampling period). For composite-level NIMI SCC and SCCI, DHI composite-SCC measurements of milk samples collected just before and after the collection of a series of 3 milk samples were retrieved for the cows enrolled in the study. Composite SCC measurements obtained from milk samples collected in the previous or in the following lactation were not considered. Likewise, composite SCC measurements obtained $\geq 50 \mathrm{~d}$ before or after the beginning or the end of the collection of a series of samples, respectively, were not retained. Finally, pairs of composite SCC measurements taken $\geq 50$ $\mathrm{d}$ apart were not considered.

NIMI SCC. Based on recommendations from Dohoo and Leslie (1991), a threshold of 200,000 cells/ $\mathrm{mL}$ was chosen to define NIMI SCC for both quarterand composite-level analyses. Only quarters or cows with SCC $<200,000$ cells $/ \mathrm{mL}$ were considered at risk of acquiring a NIMI SCC, and a NIMI SCC was deemed to have occurred whenever the following SCC measurement was $\geq 200,000$ cells $/ \mathrm{mL}$. Whenever a NIMI SCC was observed, it was deemed to have occurred halfway between the initial and subsequent SCC measurements.

Incidence Rates. For each herd and for each sampling period, quarter- and composite-level SCCI were computed as follows: for a given herd and during a given sampling period, the total number of quarters or cows experiencing a NIMI SCC was computed and 


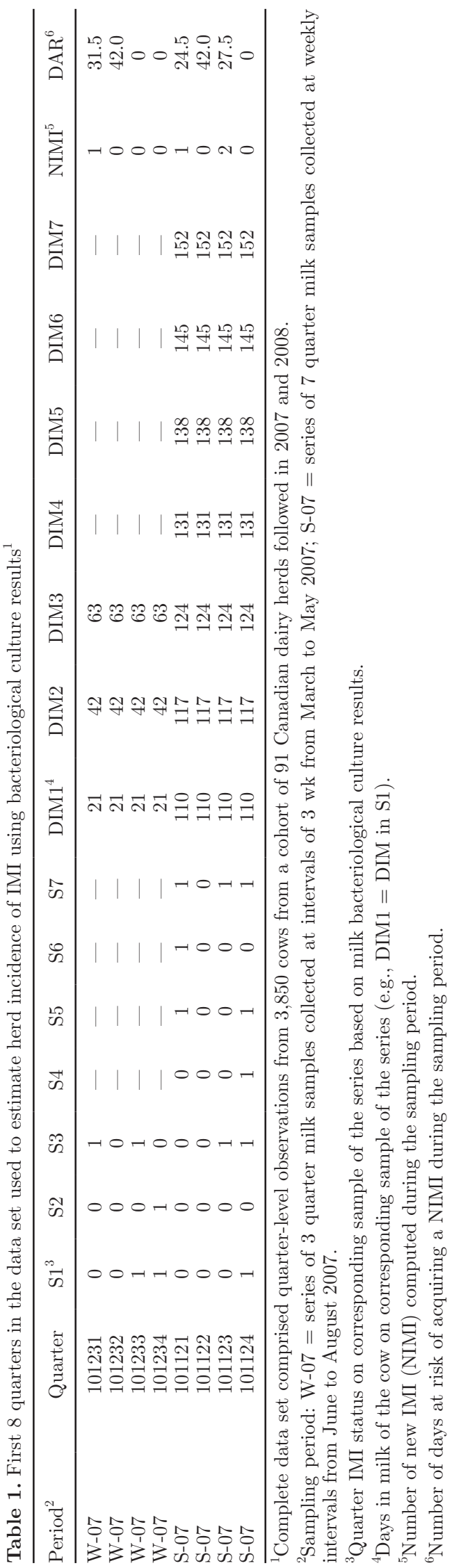

divided by the total number of quarter- or cow-days at risk of NIMI SCC acquisition. This rate was then multiplied by 30.5 to report quarter- and cow-level SCCI in NIMI SCC/quarter-month and NIMI SCC/cow-month, respectively.

\section{Statistical Analyses}

First, descriptive statistics were computed and distributions inspected for quarter- and composite-level SCCI and for major, minor, and any IMI BCI. Then, Box-Cox transformations (Box and Cox, 1964) were evaluated for their ability to improve normality of the herd IMI incidence distributions. Box-Cox transformations yielded, in general, only relatively modest improvements of the normality; IMI incidence measures were, therefore, kept on the original scale to facilitate interpretation of the results.

Relationship Between SCCI and BCI. All analyses were conducted in parallel for each category of organisms. Quarter- and composite-level SCCI were compared with major, minor, and any IMI BCI. The shape of the relationships between SCCI and BCI was first explored by visual inspection of locally weighted scatterplot smoothing curves (lowess). This relationship was then modeled using a repeated measurements structure taking into account the dependence between the multiple herd incidence measurements obtained for each herd, with a specific group of organisms BCI as dependent variable and the corresponding SCCI as explanatory variable (MIXED procedure; SAS Institute Inc., Cary, NC). For this statistical model, incidence measurements were considered uniformly spaced. Correlation structure was first evaluated using visual inspection of plots of covariance as a function of lag in time between pairs of observations. Visual inspection of these plots did not reveal any apparent seasonal effect in any of the analyses conducted. The fit of 4 different covariance structures (compound symmetry, autoregressive moving average, Toeplitz, and unstructured) was then tested. For all analyses, the unstructured correlation structure was chosen on the basis of best fit using a likelihood ratio statistic (Dohoo et al., 2009); all analyses were conducted using restricted maximum likelihood approximation as recommended by Littell et al. (2006). Whenever departure from a linear relationship was suspected based on the lowess curves visual inspection, SCCI quadratic and cubic terms were tested and retained when significant $(F-$ test, $\alpha \leq 0.05$ ).

The effect of the level of subclinical mastitis in a herd as a potential effect modifier of the relationship under investigation was then explored. To achieve this, mean herd SCS and an interaction term between SCCI 
and mean herd SCS were added to the model and the interaction term was tested for significance $(F$-test). To adjust for the numerous comparisons made, $\alpha \leq 0.01$ was chosen to determine significance of SCS as an effect modifier. The interaction term was never statistically significant and the associations under investigation were, therefore, deemed to be constant across herd subclinical mastitis levels and will not be further discussed.

Finally, measures of correlation (Spearman rho and concordance correlation coefficient) between observed and predicted rates were computed to better understand the predictive value of the SCC-derived rates. Furthermore, limits of agreement plots (plot of the difference between pairs of data against their mean) between observed and predicted rates were sketched and inspected to detect outliers and to further highlight any patterns in the predictive ability of the SCC-derived rates (Dohoo et al., 2009).

Relevance of SCCI Fluctuations over Time. Further analyses were then conducted to compare herd BCI fluctuations (i.e., changes from one sampling period to the next) over the different sampling periods (March-May 2007, June-August 2007, January-March 2008, and June-August 2008) to quarter- and composite-level SCCI fluctuations. To compare fluctuations over time, SCCI and BCI profile plots for each of the 90 herds participating to $>1$ sampling period were first inspected and visually compared. Then the SCCI and BCI differences between sample periods 2 and 1, 3 and 2 , and 4 and 3 were computed for each herd. Herd BCI fluctuations between sampling periods were then modeled as a function of herd SCCI fluctuations using a repeated measures model similar to the one previously described. Correlation structure was chosen as previously detailed and herd mean SCS was similarly evaluated as an effect modifier of the association. Again, an unstructured correlation structure was chosen, no apparent seasonal effect was noted, and herd mean SCS was not retained as an effect modifier of the association between SCCI and BCI fluctuations. Finally, measures of correlation between observed and predicted fluctuation were computed and limits of agreement plots sketched and inspected as previously described.

NIMI SCC Threshold. Finally, although the 200,000 cells/mL thresholds proposed by Dohoo and Leslie (1991) were found to be optimal to diagnose NIMI using composite sample of lactating dairy cows, other SCC thresholds could yield better results when computing herd IMI incidence or when monitoring incidence fluctuations over time. For this reason, all described analyses were also conducted using alternative SCC thresholds of 150,000 and 250,000 cells $/ \mathrm{mL}$ to define NIMI SCC.

\section{RESULTS}

Within the CBMRN database, 28,180 series of 3 milk samples were available to estimate bacteriological culture-derived and quarter SCC-derived NIMI. These sets were collected from 3,850 different cows, and 2 composite DHI SCC measurements were available for 3,556 of these cows to estimate composite SCC-derived NIMI. On average, herds were followed for 3.7 sampling periods, and a total of 336 herd-period incidence measurements could be computed. The average number of quarter-level observations used to estimate quarterlevel BCI and SCCI for a given herd and for a given sampling period was 39 for the 3 -wk sampling periods and 205 for the weekly sampling period. To compute composite-level SCCI for a given herd in a given sampling period, on average 24 pairs of DHI composite SCC measurements were available.

A complete description of the quarter-level BCI of the NCDF can be found elsewhere (Dufour et al., 2012a,b; A. M. Elmoslemany, University of Prince Edward Island, Charlottetown, PE, Canada; D. Haines, Université de Montréal, St-Hyacinthe, QC, Canada; H.W. Barkema, University of Calgary, Calgary, AB, Canada; G. Keefe, University of Prince Edward Island, Charlottetown, PE, Canada; K. Leslie, University of Guelph, Guelph, ON, Canada; D. Kelton, University of Guelph, Guelph, ON, Canada; D. T. Scholl, South Dakota State University, Brookings, SD; and I. R. Dohoo; unpublished data). Briefly, major, minor, and any IMI BCI distributions were right-skewed with median (25th and 75th percentiles) of $0.00(0.00,0.02)$, $0.13(0.05,0.28)$, and $0.17(0.08,0.36)$ NIMI/quartermonth, respectively. Quarter-level SCCI was similarly right-skewed with a median (25th and 75th percentiles) of $0.05(0.02,0.09) \mathrm{NIMI} /$ quarter-month. Compositelevel SCCI distribution was right-skewed as well with median (25th and 75 th percentiles) of $0.08(0.04,0.14)$ NIMI/cow-month.

\section{Relationship Between SCCl and $\mathrm{BCl}$}

Relationships between SCCI and BCI are illustrated in Figure 1. Linear and positive relationships could be observed between quarter-level SCCI and BCI and between composite-level SCCI and major IMI BCI. Minor or any IMI BCI, conversely, appeared to be relatively constant across composite-level SCCI values.

Quarter-Level SCC. Estimates of association between quarter-level BCI and SCCI for the different groups of organisms are reported in Table 2. Briefly, quarter-level SCCI was found to be a significant predictor of BCI for the 3 groups of organisms considered. For 
a) Major IMI vs. quarter SCC incidence

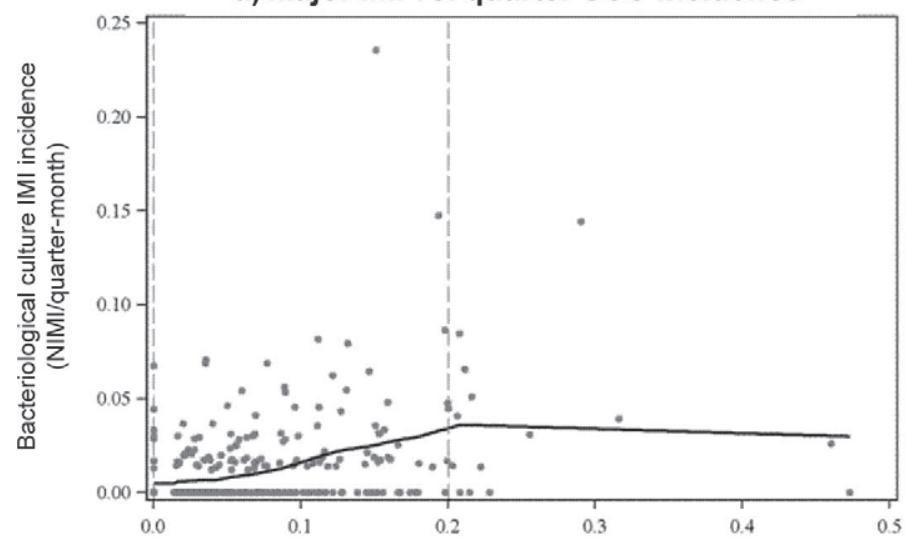

c) Minor IMI vs. quarter SCC incidence

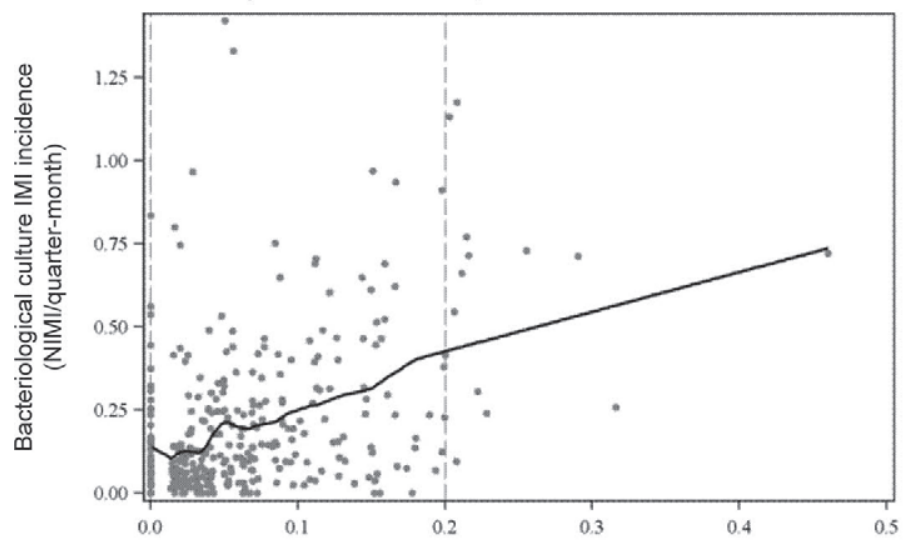

e) Any IMI vs. quarter SCC incidence

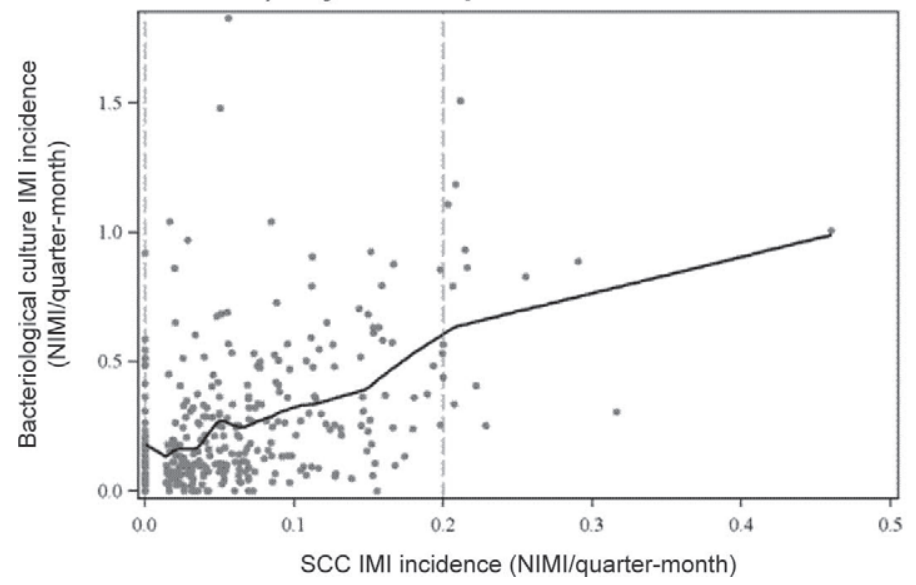

b) Major IMI vs. composite DHI SCC incidence

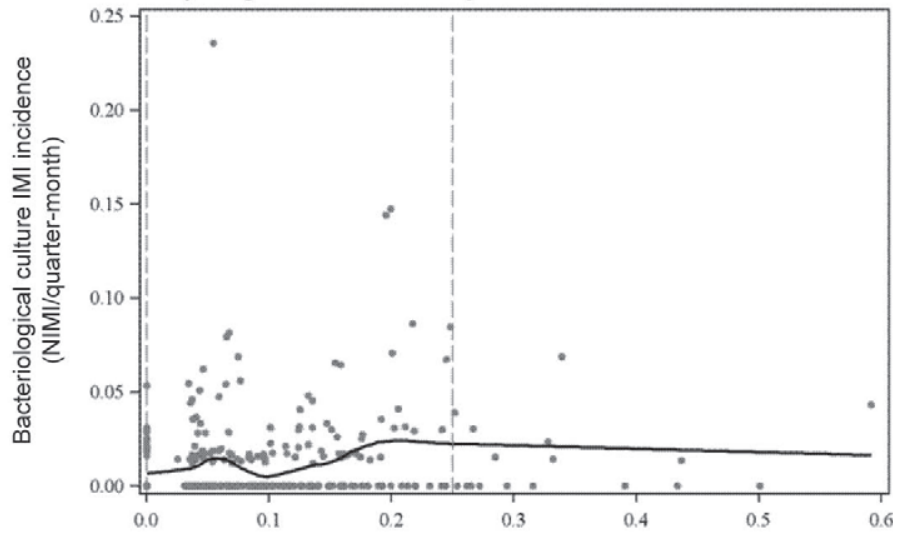

d) Minor IMI vs. composite DHI SCC incidence

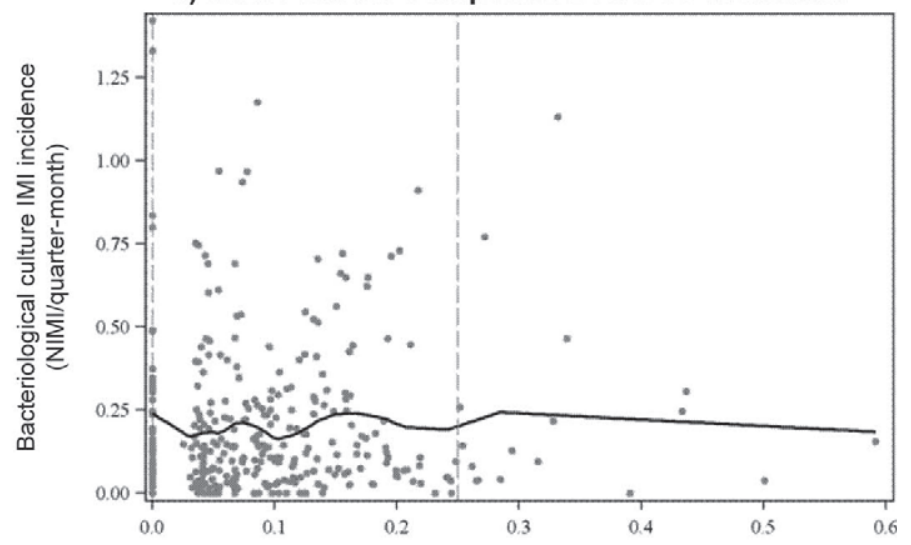

f) Any IMI vs. composite DHI SCC incidence

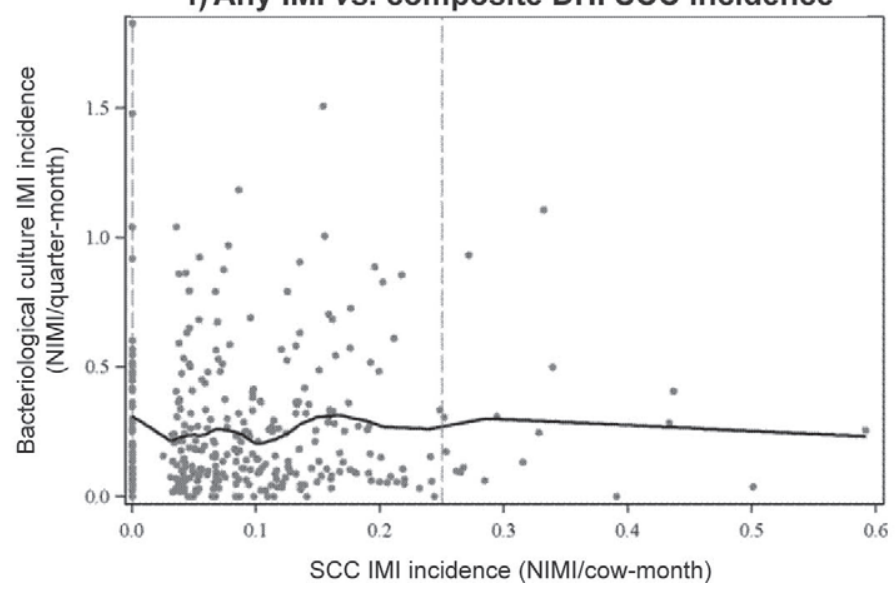

Figure 1. Scatter plots of bacteriological culture-derived herd IMI incidence (BCI) against SCC-derived herd IMI incidence (SCCI) with lowess smoothed curve, for 3 groups of organisms and for quarter and DHI composite SCC measurements; SCCI 5th and 95th percentiles are represented by vertical dashed lines. The data set comprised 336 herd-periods incidence estimates from 91 Canadian dairy herds. NIMI = new IMI.

all groups of organisms, a modest correlation between observed and predicted rates was observed, with Spearman rho ranging from 0.34 to 0.42 (Table 2). Conversely, the concordance correlation coefficient (CCC) - that also takes into consideration departure from the $45^{\circ}$ line and from the origin - indicated only a slight agreement 
between observed and predicted rates. Limits of agreement plots between observed and predicted incidence are presented in Figure 2. As can be seen, approximately $95 \%$ of predicted values fell between 0.04 units above and 0.05 units below the observed NIMI rate. For the 3 groups of organisms studied, larger absolute differences between observed and predicted incidence could be seen for herds with relatively high observed incidence (i.e., high BCI). Larger absolute major IMI incidence differences were seen, for instance, above mean BCI values of $0.03 \mathrm{NIMI}$ /quarter-month, which corresponded roughly to herd-period with observed major IMI BCI >0.06 NIMI/quarter-month. These observations, however, represented $<5 \%$ of the herd-periods studied. Similarly, larger absolute minor and any IMI incidence differences were seen above mean BCI value of $0.45 \mathrm{NIMI}$ /quartermonth, which corresponded to the small proportion of observations $(<5 \%$ for minor IMI and $<10 \%$ for any IMI) with observed minor IMI BCI $>0.70$ NIMI/ quarter-month, and observed any IMI incidence $>0.60$ NIMI/quarter-month.

Composite-Level SCC. Estimates of association between BCI and composite-level SCCI for the different groups of organisms are reported in Table 2. When computed using DHI composite data, SCCI was not a significant predictor of minor or any IMI BCI. A significant relationship, however, was observed between SCCI and major IMI BCI. The predictive ability of the composite SCC-derived incidence rate appeared to be relatively limited, however, as indicated by the low correlation between observed and predicted rates (Spearman rho: 0.14; CCC: 0.02; Table 2). Similar to the quarter SCC analyses, larger absolute differences between observed and predicted rates were observed above a mean incidence value of $0.03 \mathrm{NIMI} /$ quartermonth (Figure 2), which corresponded to the small proportion of observations $(<5 \%)$ with observed major IMI incidence $>0.06 \mathrm{NIMI} /$ quarter-month.

\section{Relevance of SCCI Fluctuations over Time}

Relationships between fluctuations of quarter- and composite-SCCI and fluctuation of BCI are illustrated in Figure 3. Positive linear relationships between quarter-level SCCI and BCI fluctuations were observed for the 3 groups of organisms. Fluctuations in BCI over time were relatively constant, however, across level of composite DHI SCC-derived incidence fluctuations. Estimates of association between quarter- and composite-SCCI fluctuations and BCI fluctuations are reported in Table 3. Fluctuation over time of the quarter SCC-derived incidence was a significant predictor of major, minor, and any IMI BCI fluctuation. For the 3 groups of organisms, a substantial correlation between observed and SCC-predicted incidence fluctuation was observed, with Spearman rho ranging from 0.54 to 0.58 . When taking into account departure from the $45^{\circ}$ line and from the origin, slight to fair agreement

Table 2. Results of 6 linear regression models on the relationship between SCC-derived (SCCI) and quarter milk bacteriological culture-derived (BCI) herd IMI incidence for 3 groups of organisms and for quarter- and composite-level SCC ${ }^{1}$

\begin{tabular}{|c|c|c|c|c|c|}
\hline $\begin{array}{l}\text { Dependent variable } \\
\mathrm{f} \text { the model }\end{array}$ & Fixed effect & Estimate & $95 \% \mathrm{CI}$ & Spearman $\rho^{2}$ & $\mathrm{CCC}$ \\
\hline \multicolumn{6}{|l|}{ Quarter-SCC analyses } \\
\hline (1) Major IMI BCI & Intercept & 0.004 & $0.002,0.006$ & 0.34 & 0.08 \\
\hline \multirow[t]{2}{*}{ (2) Minor IMI BCI } & Intercept & 0.098 & $0.075,0.121$ & \multirow[t]{2}{*}{0.38} & \multirow[t]{2}{*}{0.04} \\
\hline & Quarter $\mathrm{SCCI}^{4}$ & 0.510 & $0.217,0.803^{*}$ & & \\
\hline (3) Any IMI BCI & Intercept & 0.121 & $0.095,0.147$ & 0.42 & 0.03 \\
\hline \multirow[t]{2}{*}{ (4) Major IMI BCI } & Intercept & 0.004 & $0.001,0.006$ & \multirow[t]{2}{*}{0.14} & \multirow[t]{2}{*}{0.02} \\
\hline & Composite $\mathrm{SCCI}^{5}$ & 0.024 & $0.003,0.044 *$ & & \\
\hline \multirow[t]{2}{*}{ (5) Minor IMI BCI } & Intercept & 0.116 & $0.091,0.141$ & \multirow[t]{2}{*}{0.02} & \multirow[t]{2}{*}{0.01} \\
\hline & Composite $\mathrm{SCCI}^{5}$ & 0.025 & $-0.138,0.189$ & & \\
\hline \multirow[t]{2}{*}{ (6) Any IMI BCI } & Intercept & 0.150 & $0.121,0.179$ & \multirow[t]{2}{*}{0.02} & \multirow[t]{2}{*}{0.01} \\
\hline & Composite $\mathrm{SCCI}^{5}$ & 0.028 & $-0.163,0.219$ & & \\
\hline
\end{tabular}

\footnotetext{
${ }^{1}$ Quarter-level SCCI was compared with major, minor, and any IMI BCI; composite-level SCCI was similarly compared with major, minor, and any IMI BCI; results were obtained using repeated measures models with an unstructured correlation structure. Associations were computed using 336 herd-periods incidence estimates from 91 Canadian dairy herds.

${ }^{2}$ Spearman correlation coefficient between observed and predicted IMI incidences.

${ }^{3}$ Concordance correlation coefficient (CCC) between observed and predicted IMI incidences; CCC takes into account both precision and accuracy of the measurements (Carrasco and Jover, 2003).

${ }^{4} \mathrm{SCCI}$ in new IMI (NIMI)/quarter-month for quarter-level analyses.

${ }^{5} \mathrm{SCCI}$ in NIMI/cow-month for composite-level analyses.

*SCCI was a significant predictor $(P \leq 0.05)$ of the quarter BCI under investigation.
} 
a) Predicting major IMI incidence with quarter SCC

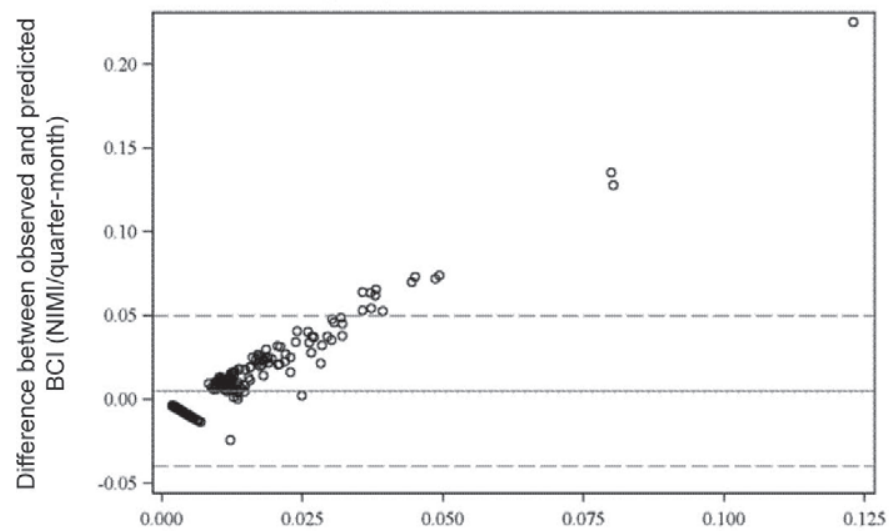

c) Predicting any IMI incidence with quarter SCC

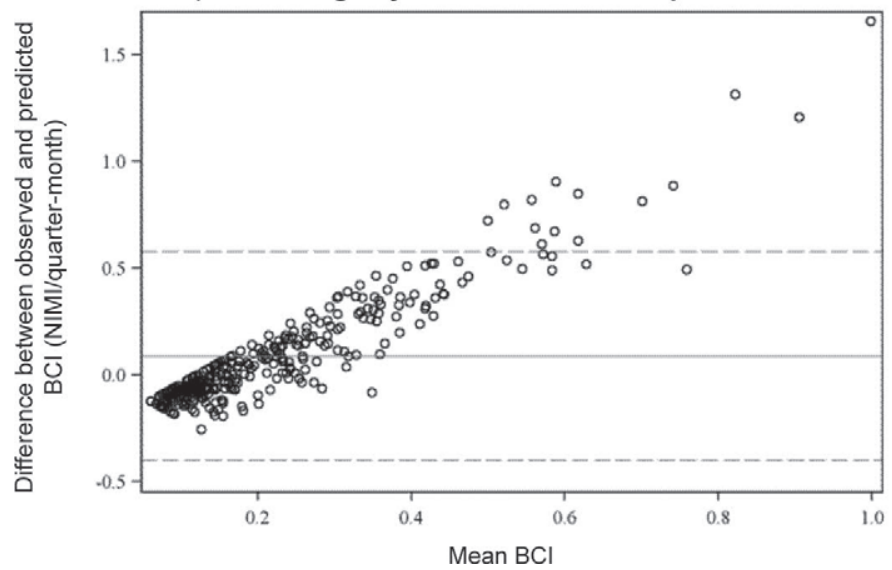

b) Predicting minor IMI incidence with quarter SCC

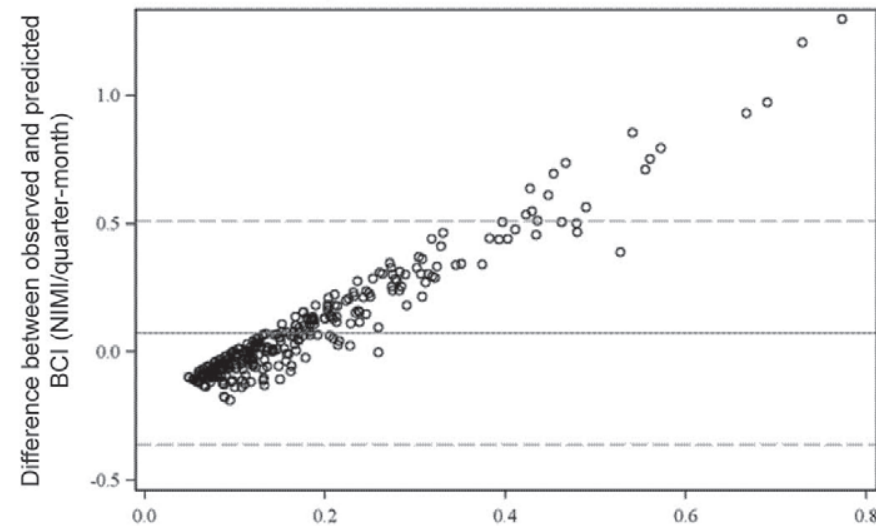

d) Predicting major IMI incidence with composite DHI SCC

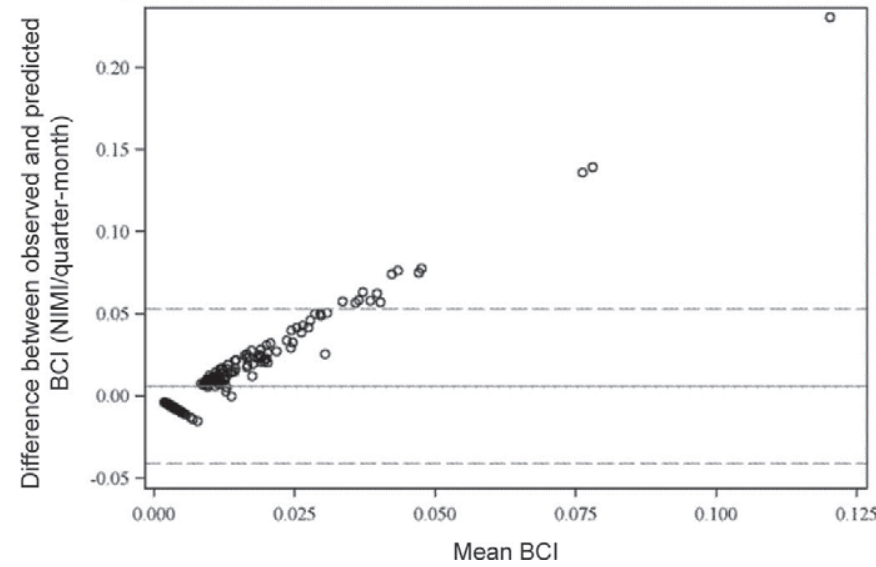

Figure 2. Limits of agreement plots between observed bacteriological culture-derived herd IMI incidence (BCI) and predicted BCI using repeated lactational SCC measurements, for different groups of organisms, and for incidence predictions based on repeated quarter and DHI composite SCC measurements. Mean incidence difference is represented by the horizontal full line. Limits of agreement lines (mean difference $\pm 1.96 \mathrm{SD})$ are represented by horizontal dashed lines. The data set comprised 336 herd-periods incidence estimates from 91 Canadian dairy herds. NIMI $=$ new IMI.

between observed and predicted incidence fluctuation was observed (CCC ranging from 0.15 to 0.28 ). Limits of agreement plots between observed and quarter SCCpredicted herd IMI incidence fluctuations are presented in Figure 4. For each of the 3 groups of organisms, the same herds exceeding the upper limit of agreement on one interval were generally found below the lower limit of agreement for the subsequent interval (data not shown), indicating that the largest absolute differences between BCI and SCCI fluctuations were potentially due to an underestimation of a relatively high BCI peak on a given sampling period.

\section{Alternative NIMI SCC Thresholds}

When using the 150,000 and 250,000 cells $/ \mathrm{mL}$ thresholds, the same significant relationships were observed, except for the composite SCCI-major IMI BCI relationship, which was statistically significant under the 200,000 cells $/ \mathrm{mL}$ threshold only. Moreover, in most situations, incidence and incidence fluctuation predictions computed using the 200,000 cells/mL threshold showed very comparable or stronger correlation with the observed values than the predictions made using the 150,000 and 250,000 cells/mL thresholds. Consequently, the use of a different SCC threshold to define NIMI appeared to be unjustified, and results from these other analyses are not presented.

\section{DISCUSSION}

Despite the widespread use of repeated SCC measurements for IMI incidence monitoring during the lactation, this is the first study to compare herd IMI incidence estimates derived from SCC to the estimates that can be derived from repeated milk bacteriological 
a) Major IMI vs. quarter SCC incidence fluctuations

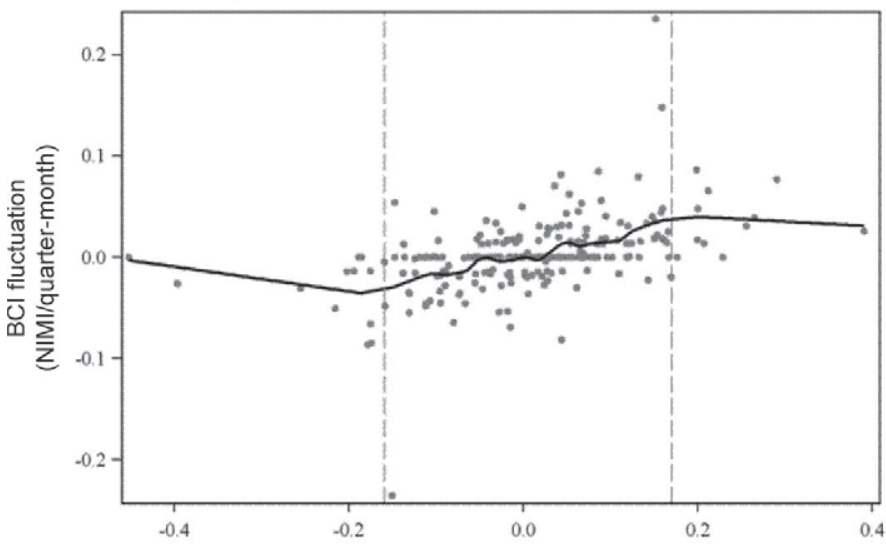

c) Minor IMI vs. quarter SCC incidence fluctuations

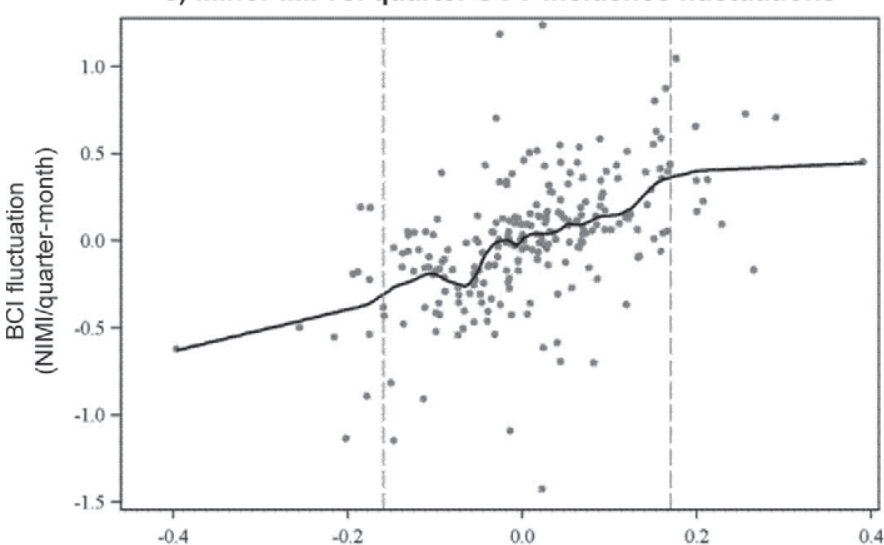

e) Any IMI vs. quarter SCC incidence fluctuations

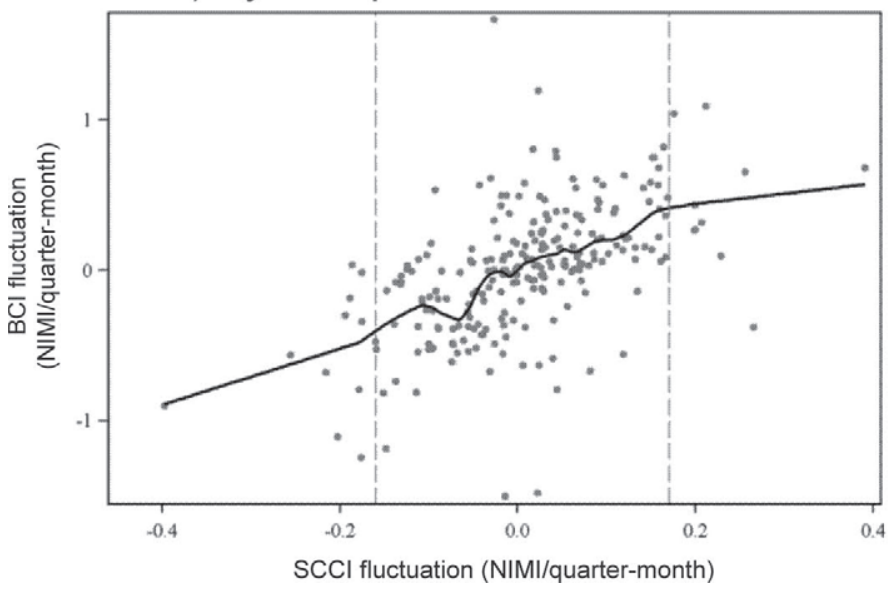

b) Major IMI vs. composite DHI SCC incidence fluctuations

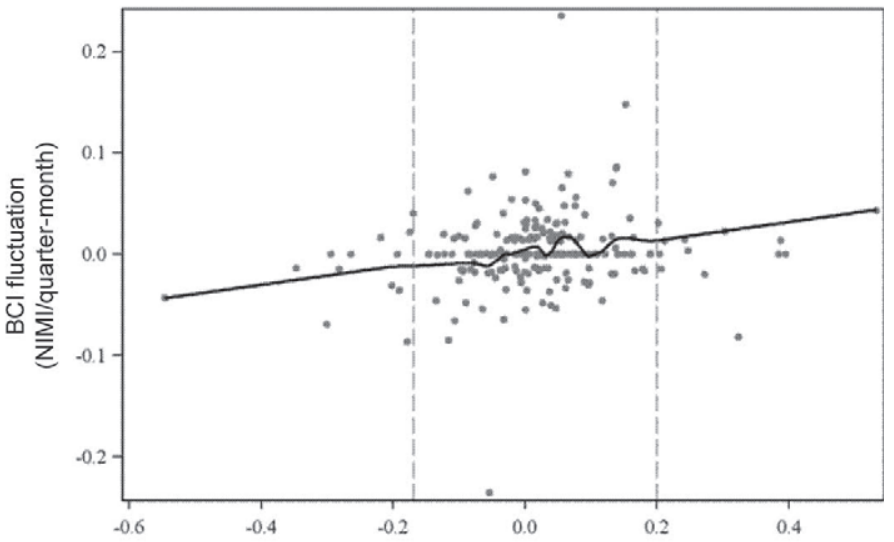

d) Minor IMI vs. composite DHI SCC incidence fluctuations

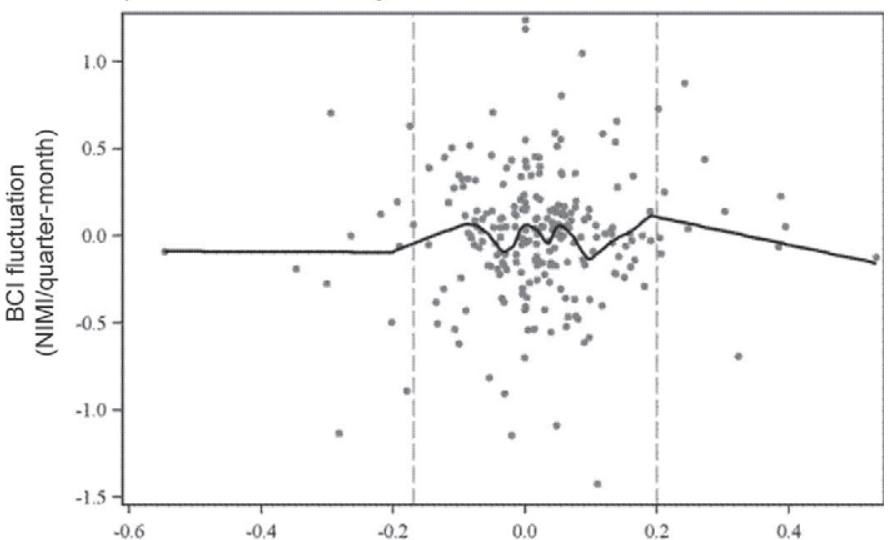

f) Any IMI vs. composite DHI SCC incidence fluctuations

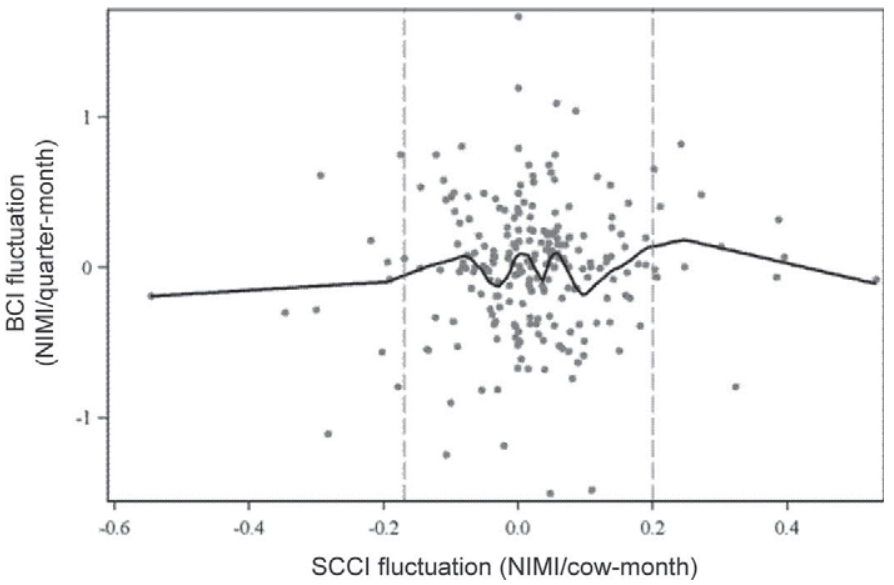

Figure 3. Scatter plots of bacteriological culture-derived herd IMI incidence (BCI) fluctuation against SCC-derived herd IMI incidence (SCCI) fluctuations with lowess smoothed curve, for 3 groups of organisms and for quarter and DHI composite SCC measurements; SCCI fluctuations 5th and 95th percentiles are represented by vertical dashed lines. The data set comprised 273 herd-periods incidence fluctuation estimates from 91 Canadian dairy herds. NIMI = new IMI.

culture. It is important to acknowledge, however, that no gold standard test exists that can be used to correctly identify IMI. Using milk bacteriological culture to define IMI and NIMI, for instance, will result in varying levels of misclassification bias depending on the pathogens studied (Dohoo et al., 2011; Dufour et al., 
Table 3. Results of 6 models on the relationship between fluctuations over time of SCC-derived (SCCI) and bacteriological culture-derived (BCI) herd IMI incidence, for 3 groups of organisms and for quarter- and composite-level SCC ${ }^{1}$

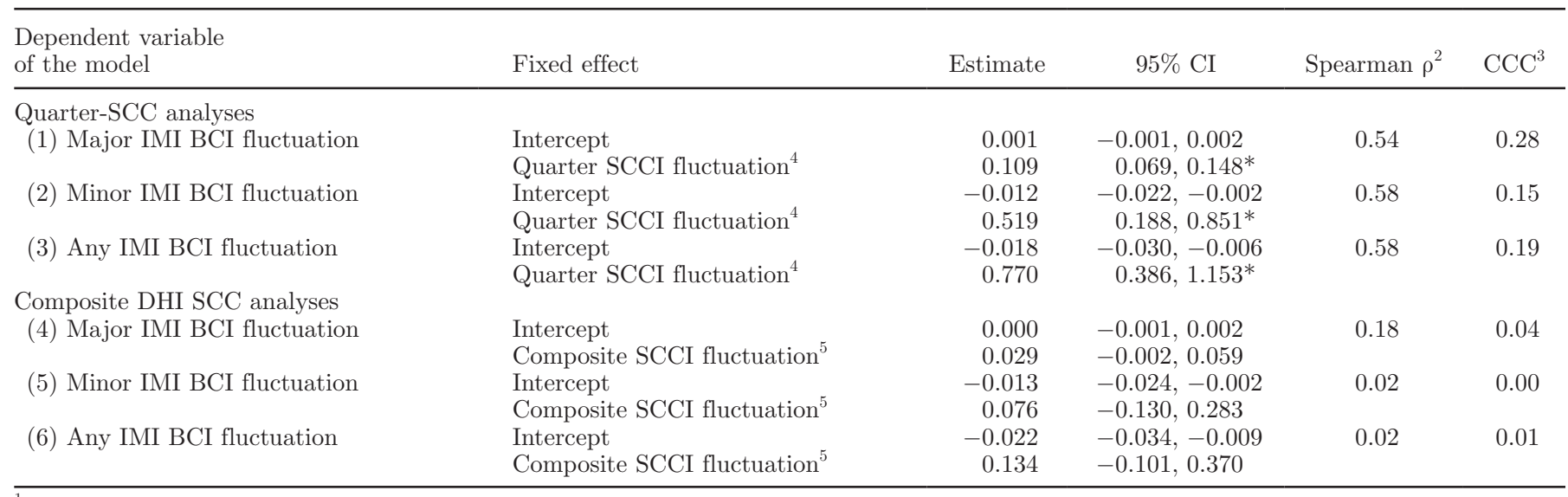

${ }^{1}$ Quarter-level SCCI fluctuation was compared with major, minor, and any IMI BCI fluctuations; composite-level SCCI fluctuation was similarly compared with major, minor, and any IMI BCI fluctuations; results were obtained using repeated measures models with an unstructured correlation structure. Associations were computed using 273 herd-periods incidence fluctuation estimates from 91 Canadian dairy herds.

${ }^{2}$ Spearman correlation coefficient between observed and predicted IMI incidence fluctuations.

${ }^{3}$ Concordance correlation coefficient (CCC) between observed and predicted IMI incidences fluctuations; CCC takes into account both precision and accuracy of the measurements (Carrasco and Jover, 2003).

${ }^{4} \mathrm{SCCI}$ fluctuation in new IMI (NIMI)/quarter-month for quarter-level analyses.

${ }^{5} \mathrm{SCCI}$ fluctuation in NIMI/cow-month for composite-level analyses.

*SCCI fluctuation was a significant predictor $(P \leq 0.05)$ of the quarter BCI fluctuation under investigation.

2012a). In the current study, the computed BCI were, therefore, likely to be biased to some extent by the imperfect sensitivity and specificity of milk bacteriological culture. Nonetheless, bacteriological culture-derived incidence estimates were used for comparison because they possibly are the only herd incidence estimates that can be obtained practically, even in research settings, on a large sample of herds.

Furthermore, the categorization of specific mastitis pathogens in the broader categories used in the current study may have biased, to some extent, the computed BCI. In the current study, several quarters that were infected with a given major pathogen at one point, and that acquired a NIMI by another major pathogen over the sampling period, were simply identified as persistently infected; they were not considered at risk of acquiring a major pathogen NIMI and were, therefore, excluded from the incidence calculation (i.e., the NIMI was not included in the incidence numerator and the quarter did not contribute any days at risk to the denominator). The resulting bias in the incidence estimates, however, would be limited for the major pathogens category, for which only 4 Staph. aureus, 2 Strep. dysgalactiae, and 2 Strep. uberis NIMI, over the 28,180 milk samples series, were excluded due to the presence of a different major pathogen at the beginning of the sampling series. For the minor pathogens category and, consequently, for the any pathogens category, the bias of the incidence estimates could be substantial.
When categorizing CNS and Corynebacterium spp. into a broader "minor pathogen" category, for instance, 217 of the 1,918 CNS NIMI were instead diagnosed as persistent minor pathogen IMI due to the presence of a Corynebacterium spp. IMI at the beginning of the sampling series; therefore, they were excluded from the minor pathogens incidence calculation. Similarly, 269 of the 812 Corynebacterium spp. NIMI were excluded from the minor pathogens incidence calculation for similar reasons.

Although a substantial bias of the minor and any pathogens BCI estimates can be expected, we could assume that the SCC-derived incidence rates would be similarly affected by the presence of quarters infected both at the beginning and end of a sampling series but by different pathogens. The SCCI-BCI relationships should, therefore, remain relatively unbiased.

In the current study, milk samples had to be frozen upon collection because of the long shipping distance to the regional laboratories responsible for the microbiological analyses and to accommodate the laboratories sample analysis capacity. Because of the low recovery rate of Mycoplasma spp. organisms from frozen milk samples (Biddle et al., 2004), and because of its low prevalence in Canada (Olde Riekerink et al., 2006), identification of Mycoplasma spp. was not carried out in the current study. Associations highlighted could, therefore, differ in areas where prevalence or incidence of Mycoplasma spp. IMI is high. 
a) Predicting major IMI incidence fluctuation with quarter SCC

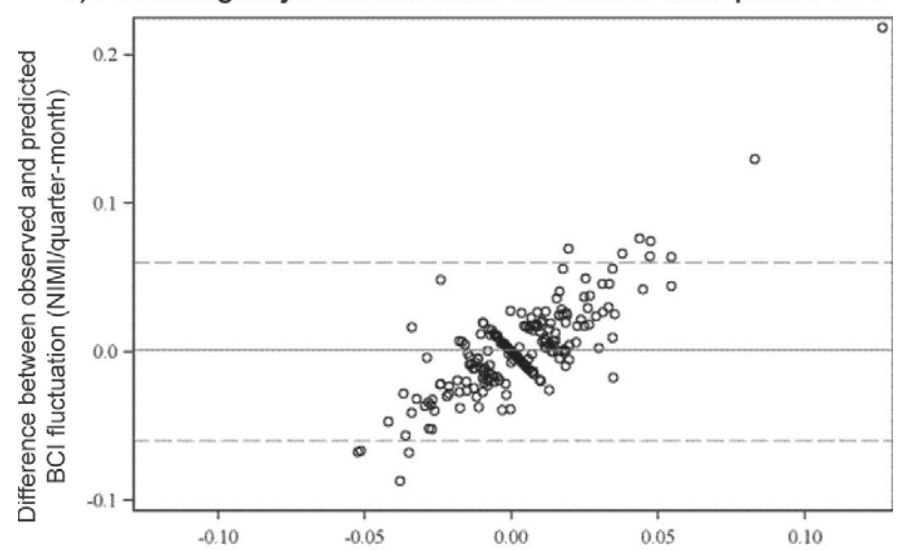

b) Predicting minor IMI incidence fluctuation with quarter SCC

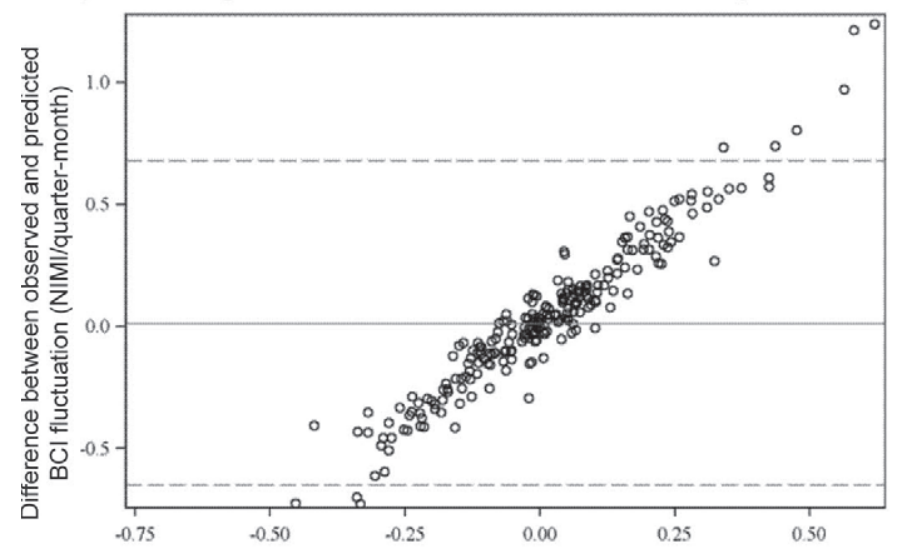

c) Predicting any IMI incidence fluctuation with quarter SCC

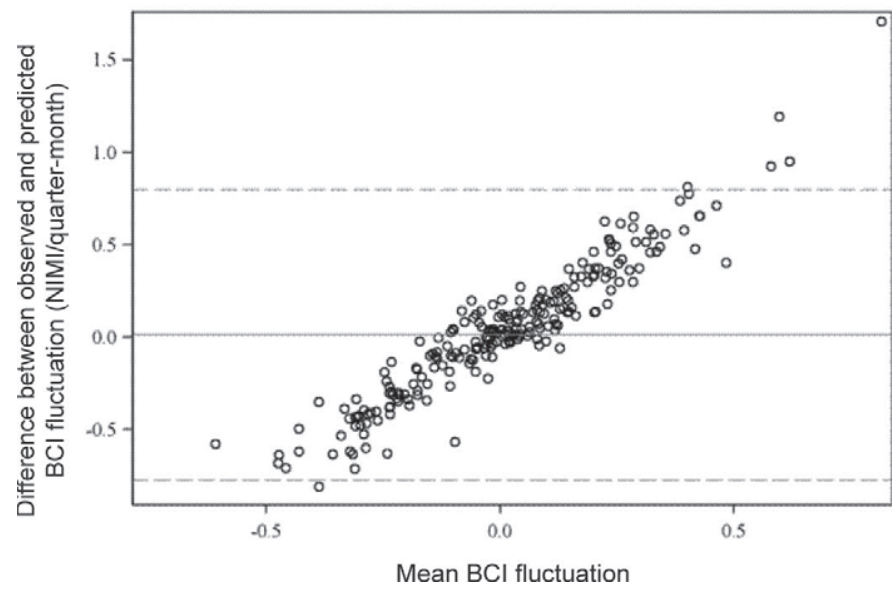

Figure 4. Limits of agreement plots between observed bacteriological culture-derived herd IMI incidence (BCI) fluctuations and predicted BCI fluctuation using repeated lactational quarter SCC measurements, for 3 different groups of organisms. Mean incidence fluctuation difference is represented by the horizontal full line. Limits of agreement lines (mean difference $\pm 1.96 \mathrm{SD}$ ) are represented by horizontal dashed lines. The data set comprised 273 herd-periods incidence fluctuation estimates from 91 Canadian dairy herds. NIMI = new IMI.
Results from the current study suggest that using the repeated quarter SCC measurements, which could be obtained using online milking system measurements, to compute a herd IMI incidence density estimate would be a valuable alternative to repeated milk bacteriological culture. Although the relationship between quarter SCC- and bacteriological culture-derived estimates showed some important departure from the $45^{\circ}$ line and from the origin, the SCC-derived rate showed a moderate correlation with the major, minor, and any IMI herd incidence rates and could potentially be used as a surrogate for these rates. For the 3 groups of organisms, however, important disagreement between observed and predicted rates was seen for the small proportion of observations with relatively high BCI (Figure 2). This inaccuracy of the quarter SCC-derived rate to diagnose herds with high $\mathrm{BCI}$ on a given sampling period is a serious issue, as these high-BCI herds are the herds that would benefit the most from being promptly identified. Moreover, this latter inaccuracy also seemed to impair, to some extent, the predictive ability of quarter SCC-derived rates for monitoring incidence fluctuations over time in a given herd. In this case, an important increase of the incidence rate may remain undetected when repeated quarter SCC measurements are used to estimate IMI incidence in place of repeated bacteriological culture. Nevertheless, in most situations, the use of repeated quarter-SCC measurements to monitor fluctuations over time of the IMI incidence rate seemed very promising as can be seen from the substantial correlation between SCC- and bacteriological culture-derived incidence fluctuations.

Conversely, results from the current study suggest that the value of using DHI-composite monthly SCC measurements as a substitute to repeated milk bacteriological culture for estimating the herd IMI incidence during the lactation may be very limited. Although composite-level SCCI was found to be a significant predictor of the major IMI BCI, correlation between observed and predicted rates was relatively low. Furthermore, fluctuation over time of composite-level SCCI was not significantly associated with BCI fluctuations, indicating that using monthly DHI composite SCC measurements for longitudinal monitoring of the IMI incidence may be impractical. These differences between repeated quarter- and composite-level SCC measurements for estimating the IMI incidence rate during the lactation are quite similar to those observed when estimating the dry period IMI incidence and elimination rates (Dufour and Dohoo, 2012). It is likely that our ability to detect the increase of SCC that follows acquisition of an IMI, especially CNS or Corynebacterium spp. IMI, in one quarter would be severely impaired by the normal SCC variations of the 3 other quarters 
of a cow. Besides, and conversely to the quarter-level SCC data and bacteriological culture results that were obtained from a single milk sample, a certain lag time was observed between the DHI-composite SCC measurements and the collection of milk samples that were used to define bacteriological culture IMI status. The DHI composite SCC measurements were obtained, on average, $17 \mathrm{~d}$ (SD: 12) before the first and $19 \mathrm{~d}$ (SD: 12) after the last sample of a 3 milk samples series. Although such a lag time may not have a very large effect on an estimate of major IMI BCI, it is likely that many of the usually short-lasting minor IMI that would be detected using repeated milk bacteriological culture over a short period may have remained undetected using repeated SCC measurements collected over a longer period. If this is the case, the lag between SCC and IMI measurements would have limited our ability to observe significant relationships between the composite-SCCI and minor and any IMI BCI in particular, and the predictive value of using composite-SCC measurement to estimate these IMI incidence rates would then be underestimated in the current study.

\section{CONCLUSIONS}

Our results support the use of repeated SCC measurements as a substitute for repeated milk bacteriological culture for computing and monitoring the incidence of IMI during the lactation, provided quarter-level SCC are available (e.g., through on-line milking system measurements). Furthermore, fluctuations over time of the IMI incidence rate that can be derived from these quarter-milk SCC measurements could be used to monitor fluctuations of the BCI and to inform further investigation and potentially modifications of the herd udder health control program. Quarter SCC-derived incidence, however, showed a more limited predictive value in herds experiencing a very high rate of IMI acquisition during a given period. This finding indicates that some important elevations of the IMI acquisition rate may remain undetected when quarter SCC-derived incidence is used to monitor herd udder health status over time. Finally, monthly composite-DHI SCC measurements could be used to compute an incidence rate that might be an acceptable substitute to the major IMI BCI derived from repeated quarter-milk bacteriological culture. The predictive value of this SCC-derived rate, however, seemed to be relatively limited and this rate appeared to be of little help for longitudinal monitoring of the herd IMI incidence.

\section{ACKNOWLEDGMENTS}

The authors thank all of the dairy producers, animal health technicians, and CBMRN regional coordinators
(Herman Barkema, University of Calgary, Canada; Trevor DeVries, University of Guelph, Canada; JeanPhilippe Roy and Luc Des Côteaux, University of Montreal, Canada; and Kristen Reyher, University of Prince Edward Island, Canada) who participated to the data collection. This research was financed by the Natural Sciences and Engineering Research Council of Canada (Ottawa, ON, Canada), Alberta Milk Edmonton, AB, Canada), Dairy Farmers of New Brunswick (Sussex, New Brunswick, Canada), Dairy Farmers of Nova Scotia (Lower Truro, NS, Canada), Dairy Farmers of Ontario (Mississauga, ON, Canada) and Dairy Farmers of Prince Edward Island (Charlottetown, PE, Canada), Novalait Inc. (Quebec City, QC, Canada), Dairy Farmers of Canada (Ottawa, ON, Canada), Canadian Dairy Network (Guelph, ON, Canada), Agriculture and AgriFood Canada (Ottawa, ON, Canada), Public Health Agency of Canada (Ottawa, ON, Canada), Technology PEI Inc. (Charlottetown, PE, Canada), Université de Montréal (Montreal, QC, Canada) and University of Prince Edward Island (Charlottetown, PE, Canada), through the Canadian Bovine Mastitis Research Network (Saint-Hyacinthe, QC, Canada).

\section{REFERENCES}

Biddle, M. K., L. K. Fox, D. D. Hancock, C. T. Gaskins, and M. A. Evans. 2004. Effects of storage time and thawing methods on the recovery of Mycoplasma species in milk samples from cows with intramammary infections. J. Dairy Sci. 87:933-936.

Box, G. E., and D. R. Cox. 1964. An analysis of transformations. J. R. Stat. Soc., B 26:211-243.

Bradley, A., and M. Green. 2005. Use and interpretation of somatic cell count data in dairy cows. In Pract. 27:310-315.

Carrasco, J. L., and L. Jover. 2003. Estimating the generalized concordance correlation coefficient through variance components. Biometrics 59:849-858.

Dohoo, I., W. Martin, and H. Stryhn. 2009. Veterinary Epidemiologic Research. 2nd ed. VER Inc., Charlottetown, Prince Edward Island, Canada.

Dohoo, I. R., and K. E. Leslie. 1991. Evaluation of changes in somatic cell counts as indicators of new intramammary infections. Prev. Vet. Med. 10:225-237.

Dohoo, I. R., J. Smith, S. Andersen, D. F. Kelton, and S. Godden. 2011. Diagnosing intramammary infections: Evaluation of definitions based on a single milk sample. J. Dairy Sci. 94:250-261.

Dufour, S., and I. R. Dohoo. 2012. Monitoring dry period intramammary infection incidence and elimination rates using somatic cell count measurements. J. Dairy Sci. 95: 7173-7186.

Dufour, S., I. R. Dohoo, H. W. Barkema, L. Des Côteaux, T. J. DeVrie, K. K. Reyher, J.-P. Roy, and D. T. Scholl. 2012a. Coagulasenegative staphylococci intramammary infection epidemiology in dairy cattle and impact of bacteriological culture misclassification. J. Dairy Sci. 95:3110-3124.

Dufour, S., I. R. Dohoo, H. W. Barkema, L. Des Côteaux, T. J. DeVrie, K. K. Reyher, J.-P. Roy, and D. T. Scholl. 2012b. Manageable risk factors associated with the lactational incidence, elimination, and prevalence of Staphylococcus aureus intramammary infections in dairy cows. J. Dairy Sci. 95:1283-1300.

Erskine, R. J. 2001. Mastitis control in dairy herds. Pages 397-433 in Herd Health: Food Animal Production Medicine. 3rd ed. Saunders, Philadelphia, PA. 
Halasa, T., K. Huijps, O. Østerås, and H. Hogeveen. 2007. Economic effects of bovine mastitis and mastitis management: A review. Vet. Q. 29:18-31.

Hogan, J. S., R. N. Gonzalez, R. J. Harmon, S. C. Nickerson, S. P. Oliver, J. W. Pankey, and K. L. Smith. 1999. Laboratory Handbook on Bovine Mastitis. National Mastitis Council, Madison, WI.

Littell, R. C., G. A. Milliken, W. W. Stroup, R. D. Wolfinger, and O. Schabenberger. 2006. SAS for Mixed Models. 2nd ed. SAS Institute Inc., Cary, NC.

Lukas, J. M., D. M. Hawkins, M. L. Kinsel, and J. K. Reneau. 2005. Bulk tank somatic cell counts analyzed by statistical process control tools to identify and monitor subclinical mastitis incidence. J. Dairy Sci. 88:3944-3952.

Madouasse, A., J. N. Huxley, W. J. Browne, A. J. Bradley, and M. J. Green. 2010. Somatic cell count dynamics in a large sample of dairy herds in England and Wales. Prev. Vet. Med. 96:56-64.

Olde Riekerink, R. G., H. W. Barkema, S. Veenstra, D. E. Poole, R. T. Dingwell, and G. P. Keefe. 2006. Prevalence of contagious mastitis pathogens in bulk tank milk in Prince Edward Island. Can. Vet. J. $47: 567-572$.

Reyher, K. K., S. Dufour, H. W. Barkema, L. Des Côteaux, T. J. Devries, I. R. Dohoo, G. P. Keefe, J. P. Roy, and D. T. Scholl 2011. The National Cohort of Dairy Farms-A data collection platform for mastitis research in Canada. J. Dairy Sci. 94:1616-1626.

Schukken, Y. H., D. J. Wilson, F. Welcome, L. Garrison-Tikofsky, and R. N. Gonzalez. 2003. Monitoring udder health and milk quality using somatic cell counts. Vet. Res. 34:579-596.

Valde, J. P., O. Østerås, and E. Simensen. 2005. Description of herd level criteria for good and poor udder health in Norwegian dairy cows. J. Dairy Sci. 88:86-92.

Zadoks, R. N., H. G. Allore, H. W. Barkema, O. C. Sampimon, G. J. Wellenberg, Y. T. Grohn, and Y. H. Schukken. 2001. Cow- and quarter-level risk factors for Streptococcus uberis and Staphylococcus aureus mastitis. J. Dairy Sci. 84:2649-2663. 